\title{
Optical methods for enabling focus cues in head-mounted displays for virtual and augmented reality
}

Hong Hua

Hong Hua, "Optical methods for enabling focus cues in head-mounted displays for virtual and augmented reality," Proc. SPIE 10219, ThreeDimensional Imaging, Visualization, and Display 2017, 102190L (10 May 2017); doi: 10.1117/12.2264157

SPIE Event: SPIE Commercial + Scientific Sensing and Imaging, 2017, Anaheim, California, United States 


\title{
Optical Methods for Enabling Focus Cues in Head-Mounted Displays for Virtual and Augmented Reality
}

\author{
Hong Hua \\ 3DVIS Laboratory, College of Optical Sciences, University of Arizona, 1630 E University Blvd, Tucson, AZ, 85721 \\ hhua@optics.arizona.edu; phone 1 520-626-8703; fax 1 520-621-4358; http://3dvis.optics.arizona.edu
}

\begin{abstract}
Developing head-mounted displays (HMD) that offer uncompromised optical pathways to both digital and physical worlds without encumbrance and discomfort confronts many grand challenges, both from technological perspectives and human factors. Among the many challenges, minimizing visual discomfort is one of the key obstacles. One of the key contributing factors to visual discomfort is the lack of the ability to render proper focus cues in HMDs to stimulate natural eye accommodation responses, which leads to the well-known accommodation-convergence cue discrepancy problem. In this paper, I will provide a summary on the various optical methods approaches toward enabling focus cues in HMDs for both virtual reality (VR) and augmented reality (AR).
\end{abstract}

Keywords: Head-mounted displays (HMD), focus cues, accommodation and convergence, virtual reality, augmented reality

\section{INTRODUCTION}

Head-mounted displays (HMD), also commonly known as near-to-eye displays (NED) or head-worn displays (HWD), have gained significant interests in recent years and stimulated tremendous efforts and resources to push the technology forward for a broad range of consumer applications [1]. For instance, a wide field-ofview (FOV), immersive HMD, which immerses a user in computer-generated virtual world or a highresolution video capture of a remote real world, is a key enabling technology to virtual reality (VR) applications. It finds a myriad of applications in gaming, simulation and training, defense, education, and other fields. Several commercial products have been recently launched for consumer applications, such as the Oculus Rift ${ }^{\circledR}$ and Samsung Gear ${ }^{\circledR}$. On the other end of the visualization spectrum, a lightweight optical seethrough HMD (OST-HMD), which enables optical superposition of digital information onto a user's direct view of the physical world and maintains see-through vision to the real world, is one of the key enabling technologies to augmented reality (AR) applications. It has been viewed as a transformative technology in the digital age. There have been tremendous investments in the commercial sectors for developing highperformance AR displays.

Despite the high promises and the tremendous progress made recently toward the development of both VR and AR displays, minimizing visual discomfort involved in wearing HMDs for an extended period remains to be an unresolved challenge. One of the key contributing factors to visual discomfort is the well-known vergence-accommodation conflict (VAC) due to the lack of the ability to render correct focus cues. The vergence cue refers to the rotation action of the eyes to bring the visual axes inward or outward to intersect at a 3D object of interest at near or far distances. The accommodation cue refers to the focus action of the eye where ciliary muscles change the refractive power of the crystalline lens and minimize the amount of blur for the fixated depth of the scene. Along with eye accommodation change, there is the retinal image blur effect where objects away from the eye's accommodation depth appear blurry in the retinal image and the degree of image blur varies with the distance of the object from the focused distance. The accommodation and retinal image blur effects together are known as the focus cues.

Three-Dimensional Imaging, Visualization, and Display 2017, edited by

Bahram Javidi, Jung-Young Son Osamu Matoba, Manuel Martínez-Corral, Adrian Stern

Proc. of SPIE Vol. 10219, 102190L - (C) 2017 SPIE - CCC code: 0277-786X/17/\$18 - doi: 10.1117/12.2264157 
In recent years, many research and development efforts have been made to address the VAC problem in HMDs and develop display methods that are capable of rendering correct or nearly correct focus cues. This paper will provide a summary of the various optical methods that aims to render correct or nearly-correct focus cues in HMD systems. This paper is adapted from a comprehensive review paper by the author published in IEEE Proceedings [2].

\section{VERGENCE-ACCOMMODATION CONFLICT}

Figure 1 schematically illustrates the naturally coupled actions of eye accommodation and convergence along with retinal blur effect when viewing a natural scene. The vergence depth of the eyes coincides with its accommodation depth and the retinal image blur of objects in the scene increases with the distances from the eye fixation point to other points at different depths. In viewing conventional HMD images, however, the stimuli for convergence and accommodation may be decoupled and conflicting. Figure 2 shows a schematic layout of a typical monocular HMD, which mainly consists of a 2D microdisplay as the image source and an eyepiece that magnifies the image rendered on the microdisplay and forms a virtual image appearing at a fixed distance from the eye. The VAC problem in HMDs stems from the fact that the image source mostly is a 2D flat surface located at a fixed distance from the eye. The conventional HMDs, regardless of being monocular or binocular, being see-through or immersive, lack the ability to render correct focus cues for the digital information which may appear at other distances than that corresponding to the virtual image plane. As a result, conventional HMDs fail to stimulate natural eye accommodation response and retinal blur effects.

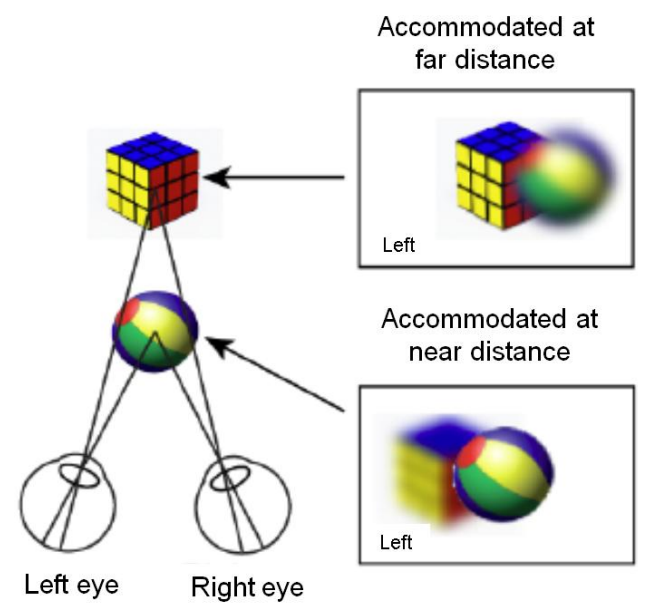

Fig. 1. Naturally coupled actions of eye accommodation and convergence in real-world view: The vergence depth of the eye where the visual axes intersect coincides with its accommodation depths and the retinal image blur of objects varies as the eye accommodation depth (cube vs. beach ball) [2].

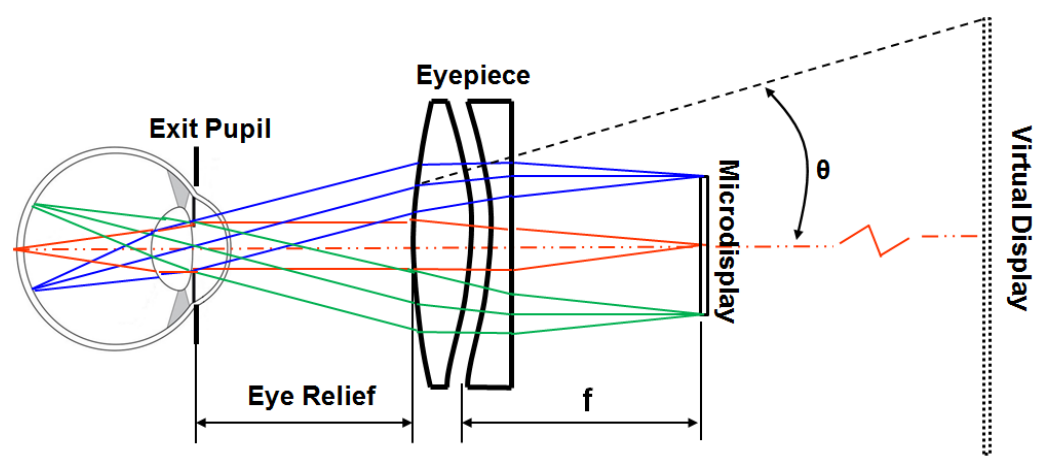

Fig. 2. Schematic layout of a conventional, monocular HMD [2]. 
Several visual cue conflicts may arise from the problem of lacking correct focus cues in HMDs [3]. One of the conflicts in a see-through AR display is the mismatch of the accommodation cues between the 2D virtual image and the real-world scene. Through a fixed-distance OST-HMD, the eye is cued to accommodate at the 2D image plane of a fixed distance for digital information. Concurrently it is also cued to accommodate and converge at the depth of various 3D objects in the natural scene. The distance gap between the display depth and real-world objects can be easily beyond what the human eyes can accommodate simultaneously. Another example of conflicts is the mismatch of accommodation and convergence between the 2D image plane and the 3D virtual world rendered in a stereoscopic 3D display (S3D). An S3D system renders pairs of images with binocular disparities on 2D flat screens at a fixed distance from the eye to create the perception of 3D virtual objects at different distances. The natural coupling of eye accommodation and convergence in viewing a real-world scene (Fig.1) is thus broken in S3D systems. When viewing the 3D virtual objects, the eyes of a user are cued to converge toward different depths of the rendered 3D contents dictated by the binocular disparity cues to fuse the stereoscopic pair while they are also cued to accommodate at the distance of the 2D display surface to bring the digital information in focus. Additionally, the VAC problem leads to a mismatch of the retinal image blur cues between the virtual and real world scenes. Virtual objects rendered via stereoscopic images, regardless of their rendered depths, are seen all in focus if the viewer accommodates on the image plane, or are seen all blurred if the user accommodate at distances separated from the image plane by distances greater than the DOF of the human eye. The retinal image blur of the virtual scene doesn't vary with the distances from an eye fixation point to other points at different depths.

Though it is not conclusive, many studies have provided strong supportive evidence that the incorrectly rendered focus cues in conventional HMDs may contribute to various visual artifacts and degraded visual performance [4-11]. In a nutshell, incorrect focus cues may contribute to the two commonly recognized issues: distorted depth perception and visual discomfort. Examples of discomfort include diplopic vision, visual fatigue, and degradation in oculomotor responses, especially after viewing such display for an extended period of time. In summary, visual discomfort is a critical concern in applications where users need to work with displays for an extended period of time.

\section{OPTICAL METHODS FOR RENDERING FOCUS CUES}

Several technical methods have been explored to address the VAC problem in conventional HMDs, attempting to approximate the visual effects created by the focus cues in natural vision. Although the classification method by Marran and Schor [3] is still valid to some extent today, to account for the recent advancements in both optical technologies and computational displays, we categorize the existing approaches into five general types: Maxwellian view displays, vari-focal plane displays, multi-focal plane (MFP) displays, integral imaging (InI) based displays, and computational multi-layer displays.

Extending the depth of field (DOF) of the virtual display is one of the simplest ways to mitigate the VAC problem in HMDs. A Maxwellian view display is one of the well-known solutions, in which a very thin bundle of rays from each field of the display is focused on the retina through a small area on the eye pupil. When the diameter of the ray projections on the pupil is substantially smaller than that of the eye pupil itself, the DOF of the display is extended and the display is considered as being accommodation free. Figure 3(a) shows the schematics of the Maxwellian viewing principle where the diverging rays from a point source $S$ is collimated by a condenser lens and then focused by an eyepiece onto the entrance pupil of the eye instead of being imaged directly on the retina [12]. To make a Maxwellian view display, a target or typically a spatial light modulator (SLM), is placed at a focal distance away from the eyepiece such that an image conjugated to the SLM is formed on the retina. Due to the fact that each field on the SLM is imaged through a narrow aperture on the pupil (pinhole), the image is observed with very large DOF without requiring eye accommodation. For this reason, the Maxwellian view display may be characterized as the pinhole optics approach [3]. Several efforts have been made to develop this type of displays aiming to address the VAC 
problem. A Maxwellian view retinal projection display was proposed by Ando et al. for accommodation-free HMDs [13], where a collimated laser source was utilized to illuminate the pixels of a SLM and is focused by a holographic optical element (HOE) at the center of the eye pupil. As a result, the image pattern shown on the SLM is directly imaged on the retina without eye accommodation. Two optical see-through bench prototypes were demonstrated, one with a reflective DMD and one with a transmissive LCD as the SLM, along with a holographic optical element as the imaging lens [13]. Both prototypes demonstrate a large DOF for the virtual display due to the benefit of the Maxwellian view. Instead of using coherent laser source [14], Von Waldkirch et al. demonstrated a retinal projection display prototype based on Maxwellian view in which a partially coherent light emitting diode (LED) illuminates an LCD through a condenser lens. Yuuki et al. demonstrated an implementation of a dense Maxwellian view display which is composed of a regular base display such as a LCD panel, a light absorption layer with pinhole patterns, and a plano-convex fly-eye lens sheet [15]. Maimone et al. demonstrated a compact, wide-FOV OST-HMD design, namely Pinlight displays [16] and a prototype display in the form factor of large glasses was demonstrated, offering a $110^{\circ}$ diagonal FOV.

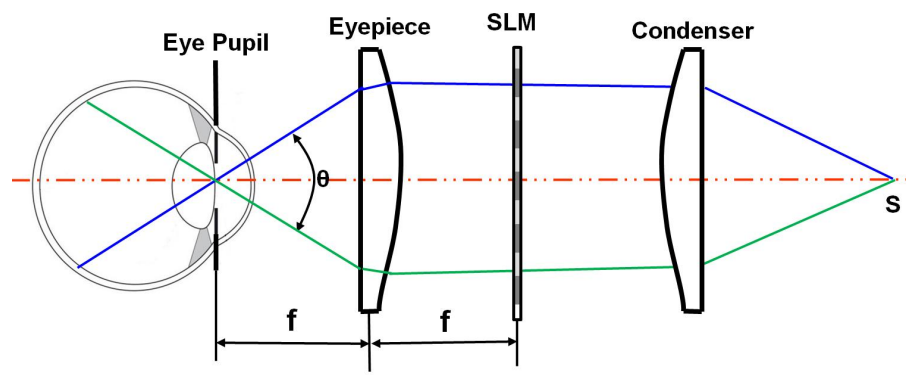

Fig. 3. Schematic illustration of a Maxwellian view display [2].

Another simple remedy to the VAC problem and facilitating proper cue for eye accommodation response is the vari-focal approach, as illustrated in Fig. 4(a), which dynamically compensates the focal distance, Z, of a single-plane display to match it with the convergence depth of the eye. The focus compensation may be achieved by a mechanical mechanism or an electronic-driven active optical element. The mechanical mechanism may be implemented by mechanically zooming the eyepiece of the display [17] or adjusting the distance between the micro-display and the eyepiece [18]. Instead of zooming the eyepiece focus through mechanically adjustable parts, a range of electronic-driven active optical elements can be used [19]. The varifocal method typically requires dynamically tracking the eye convergence distance in real time. It is able to render correct focus cues for the fixated objects if the focal distance of the virtual display is in synchrony with the distance of eye fixation, but objects at other depths would still have incorrect focus cues. The vari-focal method may be further improved by applying a depth-dependent blur filter to simulate the retinal image blur and may improve depth perception to some extent. Liu and Hua demonstrated the first OST-HMD prototype with focus control using an eyepiece design integrated with a liquid lens, for which the optical layout is shown in Fig. 4(b) [19]. The prototype is capable of dynamically controlling the focal distance of the display from 5 to 0 diopters (the near point of the eye to infinity) by driving the liquid lens from $38 \mathrm{Vrms}$ to $51 \mathrm{Vrms}$, offering a diagonal FOV of about $28^{\circ}$. The system was further extended by integrating a binocular eyetracking system which enables tracking the eye convergence depth of the viewer, addressing the focal distance of virtual image according to the vergence depth, and rendering gaze-contingent retinal blur cues in real time. Experiments to assess the depth perception and eye accommodative response to a vari-focal display technique have been demonstrated [20]. The results suggest the potentially improved depth perception induced by appropriately rendered focus cues, in comparison to findings on traditional S3D displays, which suggest distorted and compressed perception of depth. 


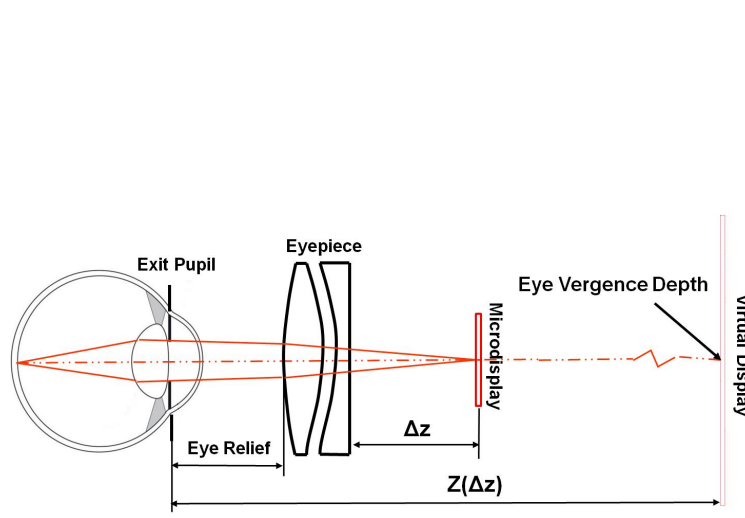

(a)

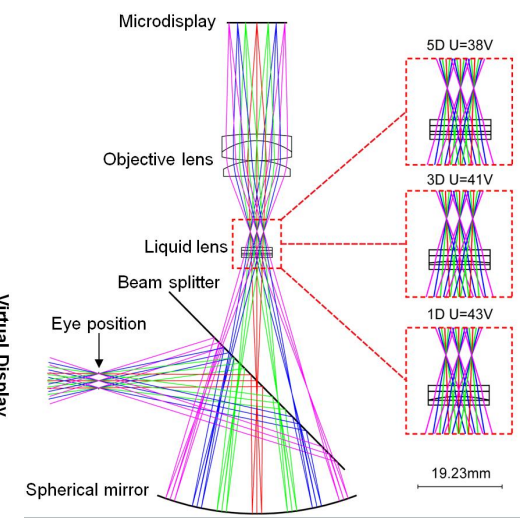

(b)

Fig. 4. (a) Schematicl layout of vari-focal HMD method [2]; (b) optical layout of a vari-focal OSTHMD design using a liquid lens [19].

Multi-focal plane (MFP) displays create a stack of discrete focal planes dividing an extended 3D scene volume into multiple zones along the visual axis. Through these focal planes, they additively reconstruct the light fields of a large scene volume by sampling its projections at different depths. An MFP-based HMD can be thought of sampling the light field projections of a 3D scene at different depths along the visual axis and the stacks of the projections additively render the light field of the scene seen from a fixed viewpoint. Unlike a vari-focal method, the MFP method does not require a mechanism to determine the point of vergence and is capable of rendering correct focus cues through a 3D volume. Fig. 5(a) shows a schematic diagram of a dualfocal plane example in which the projections of a 3D donut and a sphere are rendered by FPI and FPII, respectively. Additionally, the projections of the objects rendered by the front layer FPI can be rendered as occlusion masks on FPII (e.g. the projection of the donut on FPII) to render correct occlusion relationships among these objects. Multi-focal planes may be implemented either by spatially multiplexing a stack of 2D displays [21-24] or by rapidly switching the focal distance of a single 2D display sequentially by a high-speed vari-focal element (VFE) in synchronization with the frame rendering of multi-focal images (i.e. in a timemultiplexed fashion) [25-28]. For instance, among the spatial-multiplexing implementations, Akeley et al. demonstrated the first experimental implementation of a spatially-multiplexed three-focal plane display intended for HMD applications with an equal spacing of 0.67 diopters between adjacent focal planes [22]. The prototype covers a fixed depth range from $0.311 \mathrm{~m}$ to $0.536 \mathrm{~m}$ by dividing a flat panel display into three focal planes through 3 beamplitters placed at different distance to the viewer. More recently, Schowengerdt et al. suggested a spatial-multiplexing retinal scanning display by utilizing a fiber array to produce multi-focal bundle of beams [23]. Similarly, several time-multiplexing MFP prototypes were demonstrated over the last decade. McQuaide et al [25] demonstrated a dual-focal plane retinal scanning display in which a 2D image is generated on a pixel-by-pixel basis by raster scanning a modulated laser beam across the visual field and the focus cues of the pixels are rendered by defocusing the laser beam through a deformable membrane mirror device. Using a liquid lens as the VFE and an OLED microdisplay as the image source, Liu and Hua demonstrated the first prototype of a dual-focal plane optical see-through AR display, which maintains nonobstructive see-through view to the real world [27]. Love et al demonstrated a prototype with four fixed focal planes generated through birefringent lenses as the VFEs and high refresh rate CRTs as the image sources [28]. In general, the spatial-multiplexed approach allows for rendering multiple focal planes in parallel and reduces the speed requirements for the display technology. On the other hand, its practical implementation is challenged by the lack of stack display technologies with high transmittance and by the demand for computational power to simultaneously render a stack of 2D images of a 3D scene. The time-multiplexed approach at a flicker-free rate demands for high response speed for the active optical element, the display 
device, and the graphics rendering engine and the response speed is proportional to the number of flicker-free focal planes to be portrayed.

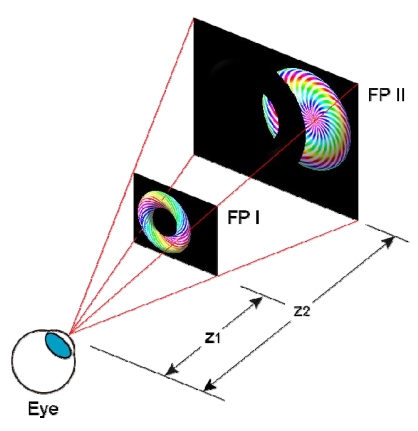

(c)

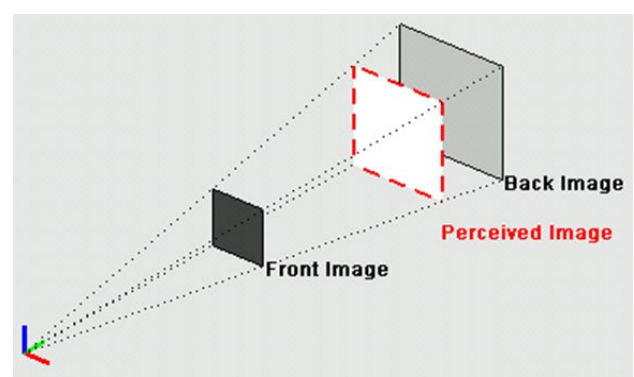

(b)

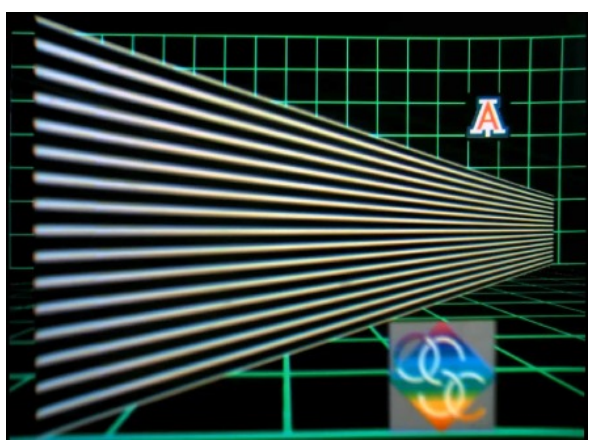

(c)

Fig. 5. Schematic illustration of (a): multi-focal plane display; (b) Depth-fused multi-focal plane display; and (c) Photograph example of a six-focal-plane depth-fused multi-focal plane display [31].

To achieve good image quality with a significantly reduced number of focal planes, the conventional multifocal plane approach may be further improved by incorporating a depth-fused 3D (DFD) perception, where two overlapped images displayed on two transparent screens at two different depths may be perceived as a single-depth image, as illustrated in Fig. 5(b). The perceived depth of the fused image may be manipulated by modulating the luminance ratio between the two images through depth-weighting fusing function to optimize the contrast magnitude and gradient of the fused image which create cues for stimulating and stabilizing the eye accommodation responses [29-31]. A multi-focal plane display incorporating the DFD effect is thus referred to as depth-fused multi-focal plane displays, or DFD-MFP displays. This method significantly reduces the number of necessary focal planes to an affordable level to render correct or nearly-correct focus cues for 3D objects spanning in a large depth range to the viewer [29-31]. Hu and Hua recently demonstrated six-focal plane OST-HMD prototypes. Fig. 5(c) shows an example image captured from the prototype system where the scene was depth-fused via 6 discrete focal planes placed at $0,0.6,1.2,1.8,2.4$, and 3 diopters, respectively [30, 31].

Integral imaging based displays reconstruct the light fields of a 3D scene by angularly sampling the directions of the light rays apparently emitted by the 3D scene and viewed from different eye positions [34-38]. As illustrated in Fig. 6(a), a simple InI-based display typically consists of a display panel and a 2D array which can be a micro-lens array (MLA) [37] or pinhole array [38]. The display renders a set of 2D elemental images, each of which represents a different perspective of a 3D scene. The conical ray bundles emitted by the corresponding pixels in the elemental images intersect and integrally create the perception of a 3D scene that appears to emit light and occupy the 3D space. The InI-based display using 2D arrays allows the reconstruction of a 3D shape with full-parallax information in both horizontal and vertical directions, which is its main difference from the conventional autostereoscopic displays with only horizontal parallax using onedimensional parallax barriers or cylindrical lenticular lenses. Lanman et al demonstrated a non-see-through light field display by directly placing a microlens array (MLA) and an OLED microdisplay in front of the eyes to render the light field of a 3D scene for VR applications [36]. Hua and Javidi demonstrated the first practical implementation of an OST-HMD design that integrates a microscopic InI (micro-InI) method for full-parallax 3D scene visualization with the emerging freeform optical technology for OST-HMD eyepiece optics [37]. This approach enables a compact 3D integral imaging OST-HMD (InI-OST-HMD) with fullparallax light field rendering capability. Fig. 6(b) shows the schematic optical layout of an InI-OST-HMD system. The optics consists of a micro-InI unit, a wedge-shaped freeform eyepiece, and a see-through freeform corrector lens. The micro-InI unit, consisting of a high-resolution micro-display and a MLA, reproduces the full-parallax light fields of a 3D scene, which replaces a conventional 2D microdisplay as the 
image source. The freeform eyepiece optics directly relays the reconstructed 3D light fields into a viewer's eye for viewing. In the see-through path, a freeform corrector lens is cemented with the freeform prism and optically enables non-obtrusive view of the real world scene.

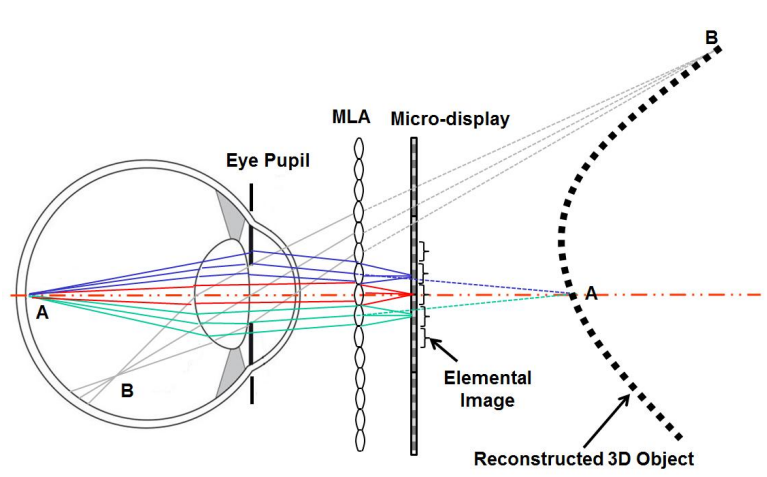

(a)

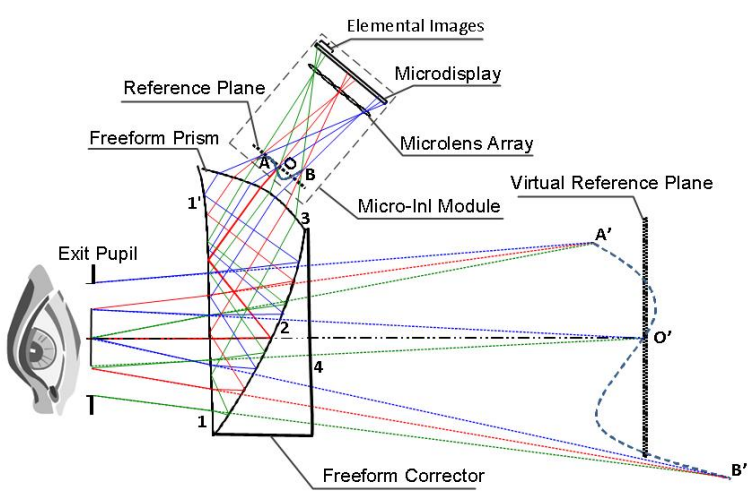

(b)

Fig. 6. (a) Schematic illustration of an integral imaging based display method [2]; (b) optical layout of an InIOST-HMD design [37].

Instead of using the InI-based architecture to angularly sample the directional light rays apparently emitted by a 3D scene through a pinhole array or MLA, a computational multi-layer light field display samples the directional rays through multi-layers of pixel arrays. As illustrated in Fig.7, it typically consists of multiple layers of SLMs illuminated by either a uniform or directional backlight [39-41]. The light field of a 3D scene is computationally decomposed into a number of masks representing the transmittance of each layer of the light attenuators. The intensity value of each light ray entering the eye from the backlight is the product of the pixel values of the attenuation layers which the ray intersects. It shares similarity to the MFP-based and the InI-based display methods. Unlike the additive nature of the MFP approach to light field rendering, the multiple SLM layers in a computational display method typically operates in a multiplicative fashion. Combining with the backlight, they aim to angularly sample the directions of the light rays apparently emitted by a 3D scene in a fashion similar to the InI-based display. Maimone and Fuchs pioneered the work to apply the multilayer computational light field display technique for its usage in HMDs and demonstrated the first computational multilayer AR display [40]. More recently, Wetzstein et al. extended their multi-layer factored light field autostereoscopic display method and demonstrated a light field stereoscope for immersive VR applications [41].

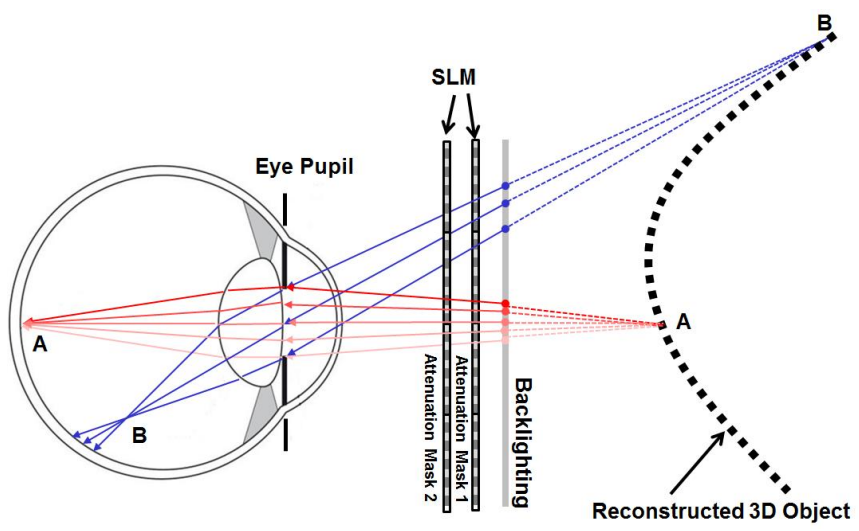

Fig. 7. Schematic layout of computational multi-layer display where the dotted curve illustrates the reconstructed 3D shapes [2]. 


\section{CONCLUSION}

This paper summarized the various optical methods that have been explored to address the VAC problem in HMD systems. Among the five methods summarized above, the Maxwellian view display attempts to extend the DOF of the virtual display to the extent such that eye accommodation is no longer required to view the virtual scene. This allows the eye to accommodate and converge freely to view real-world scenes, without compromising the image sharpness of the virtual display, which partially resolves the vergenceaccommodation conflict in OST-HMDs. However, it is unable to produce natural retinal blur cues to virtual scenes. The vari-focal approach attempts to adapt the focus of the display in real-time to the vergence depth corresponding to the region of interest. It can be a simple and effective modification to conventional HMDs and is able to address the VAC problems to a great extent, but it is unable to produce natural retinal blur cues. The MFP, integral-imaging, and multi-layer approaches are commonly referred to be light field displays, which render a true $3 \mathrm{D}$ scene by sampling either the projections of the scene at different depths or the directions of the light rays apparently emitted by the scene and viewed from different eye positions. The MFP method is an extension to the bifocal lens method described earlier in [5] by providing a larger number of focal plane sampling and 3D continuity. Both the InI-based method and the computational multi-layer displays share great similarities to the pinhole optics solution.

\section{ACKNOWLEDGEMENT}

This work is partially supported by the National Science Foundation award IIS-14-22653. Dr. Hong Hua has a disclosed financial interest in Magic Leap Inc. The terms of this arrangement have been properly disclosed to The University of Arizona and reviewed by the Institutional Review Committee in accordance with its conflict of interest policies.

\section{REFERENCES}

[1] J. P Rolland, and H. Hua, "Head-mounted display systems," in Encyclopedia of Optical Engineering (Editors: R. Barry Johnson and Ronald G. Driggers), New York, NY: Marcel Dekker, pp. 1-13, 2005.

[2] H. Hua, "Enabling focus cues in head-mounted displays," Proceedings of the IEEE, 105(5):805-824.

[3] L. Marran and C. Schor, "Multiaccommodative stimuli in VR Systems: Problems \& Solutions," Human Factors, 39(3), 382-388, 1997.

[4] T. Inoue and H. Ohzu, "Accommodative Responses to Stereoscopic Three-Dimensional Display," Applied Optics, 36(19), 4509-4515, 1997.

[5] C. Vienne, L. Sorin, L. Blonde, Q. Huynh-Thu, P. Mamassian, "Effect of the accommodationvergence conflict on vergence eye movements," Vision Research, 100(2014) 124,133, (2014).

[6] T. Takeda, K. Hashimoto, N. Hiruma, and Y. Fukui, "Characteristics of accommodation toward apparent depth," Vision Res., 39(12), pp. 2087-2097, 1999.

[7] J.P. Wann, S. Rushton, and M. Mon-Williams, "Natural Problems for Stereoscopic Depth Perception in Virtual Environments," Vision Research, 35(19), 2731-2736, 1995.

[8] S.J. Watt, K. Akeley, M.O. Ernst, and M.S. Banks, "Focus Cues Affect Perceived Depth,” J. Vision, 5(10), 834-862, 2005.

[9] P. Howarth, "Potential hazards of viewing 3-D stereoscopic television, cinema, and computer games: a review," Ophthalmic and Physilogical Optics, 31, pp 111-122, 2011.

[10] D.M. Hoffman, A.R. Girshick, K. Akeley, and M.S. Banks, "Vergence-Accommodation Conflicts Hinder Visual Performance and Cause Visual Fatigue,” J. Vision, 8(3), 1-30, (2008). 
[11] K. Ukai and P.A. Howardth, "Visual fatigue caused by viewing stereoscopic motion images: background, theories, and observations," Displays, 29(2), pp. 106-116, 2008.

[12] G. Westheimer, "Maxwellian viewing system," Vision Res. 6,pp. 669-82, 1966.

[13] T. Ando and E. Shimizu, "A head-mounted display using a holographic optical element," in Threedimensional television, video, and display technologies, B. Javidi and F. Okano, Eds. Springer Science \& Business Media, pp. 67-100, 2002.

[14] M. von Waldkirch, P. Lukowicz, and G. Tr"oster, "LCD-based coherent wearable projection display for quasi accommodation-free imaging," Opt. Commun. 217, 133-140, 2003.

[15] A. Yuuki, K. Itoga, T. Satake, "A new Maxwellian view display for trouble-free accommodation," Journal of the SID, 20(10: 581-588, 2012.

[16] A. Maimone, D. Lanman, K. Rathinavel, K. Keller, D. Luebke, H. Fuchs, "Pinlight displays: wide field of view augmented reality eyeglasses using defocused point light sources," ACM Trans. On Graphics, 33(4) 89:1-11, 2014.

[17] S. Shiwa, K. Omura, and F. Kishino, "Proposal for a 3-D display with accommodative compensation: 3DDAC", Journal of the SID, 4(4), pp. 255-261, 1996.

[18] T. Shibata, T. Kawai, K. Ohta, M. Otsuki, N. Miyake, Y. Yoshihara, and T. Iwasaki, "Stereoscopic 3-D display with optical correction for the reduction of the discrepancy between accommodation and convergence," Journal of SID, 13(8), pp. 665-671, 2005.

[19] S. Liu, D. Cheng, and H. Hua, "An optical see-through head-mounted display with addressable focal planes," in Proc. of IEEE and ACM International Symposium on Mixed and Augmented Reality (ISMAR 2008), 2008.

[20] S. Liu, H. Hua, and D. Cheng, "A novel prototype for an optical see-through head-mounted display with addressable focus cues.," IEEE Trans. Vis. Comput. Graph. 16, 381-393, 2010.

[21] J. P. Rolland, M. Kureger, and A. Goon, "Multifocal planes head-mounted displays," Applied Optics, 39(19), pp. 3209-14, 2000.

[22] K. Akeley, S. J. Watt, A. R. Girshick, and M. S. Banks, "A Stereo Display Prototype with Multiple Focal Distances," ACM Trans. Graph. 23, 804-813, 2004.

[23] B. T. Schowengerdt, M. Murari, E. J. Seibel, "Volumetric display using scanned fiber array," SID Symposium Digest of Technical Papers, 2010.

[24] D. Cheng, Q. Wang, Y. Wang, G. Jin, "Lightweight spatial-multiplexed dual focal-plane headmounted display using two freeform prisms," Chinese Optics Letters, 11(3):031201, 2013.

[25] S. C. McQuaide, E. J. Seibel, J. P. Kelly, B. T. Schowengerdt, and T. A. Furness, "A retinal scanning display system that produces multiple focal planes with a deformable membrane mirror," Displays, 24(2), pp. 65-72, 2003.

[26] S. Suyama, M. Date, and H. Takada, "Three Dimensional Display System with Dual Frequency Liquid Crystal Varifocal Lens,” Jpn. J. Appl. Phys. 39(2000): 480-484, 2000.

[27] S. Liu and H. Hua, "Time-multiplexed dual-focal plane head-mounted display with a fast liquid lens," Optics Letter, 34(11):1642-44, 2009.

[28] G. D. Love, D. M. Hoffman, P. J. W. Hands, J. Gao, A. K. Kirby, and M. S. Banks, "High-speed switchable lens enables the development of a volumetric stereoscopic display," Opt. Express 17(18), 15716-25, 2009.

[29] S. Liu and H. Hua, "A systematic method for designing depth-fused multi-focal plane threedimensional displays," Opt. Express, 18(11), 11562-11573, 2010.

[30] X. Hu and H. Hua, "Design and Assessment of a Depth-Fused Multi-Focal-Plane Display Prototype," Disp. Technol. J. PP, 1, 2014.

[31] X. Hu and H. Hua, "High-resolution optical see-through multi-focal-plane head-mounted display using freeform optics," Optics Express, 22(11): 13896-13903, 2014.

[32] S. Ravikumar, K. Akeley, and M. S. Banks, "Creating effective focus cues in multi-plane 3D displays,” Opt. Express 19, 20940-20952, 2011. 
[33] X. Hu and H. Hua, "Design and tolerance of a free-form optical system for an optical see-hrough multi-focal-plane display," Applied Optics, 54(33): 9990-9, 2015.

[34] G. M. Lippmann, "Epreuves Reversibles Donnant la Sensation du Relief," J. Phys. 7, 4th series, 821$825,1908$.

[35] X. Xiao, B. Javidi, M. Martinez-Corral, and A. Stern , "Advances in Three-Dimensional Integral Imaging: Sensing, Display, and Applications,” Applied Optics, 52(4):. 546-560, 2013.

[36] D. Lanman and D. Luebke, "Near-eye light field displays, " Proc. ACM SIGGRAPH (ACM Transaction on Graphics), 2013.

[37] H. Hua and B. Javidi, "A 3D integral imaging optical see-through head-mounted display“, Optics Express, 22(11): 13484-13491, 2014.

[38] W. Song, Y. Wang. D. Cheng, Y. Liu, "Light field head-mounted display with correct focus cue using micro structure array," Chinese Optics Letters, 12(6): 060010, 2014.

[39] G. Wetzstein, D. Lanman, M. Hirsch, and R. Raskar, "Tensor Displays: Compressive light field synthesis using multilayer displays with directional backlighting," Proc. ACM SIGGRAPH (ACM Transaction on Graphics), 2012.

[40] A. Malmone, and H. Fuchs, "Computational augmented reality eyeglasses," Proc. of ISMAR, pp. 2938, 2013.

[41] F. Huang, K. Chen, G. Wetzstein. "The Light Field Stereoscope: Immersive Computer Graphics via Factored Near-Eye Light Field Displays with Focus Cues", ACM SIGGRAPH (Transactions on Graphics), 33(5), 2015. 